\title{
Programming of the Generalized Nonlinear Paraxial Equation for the Formation of Solitons with Mathematica
}

\author{
Frederick Osman and Robert Beech \\ School of Quantitative Methods and Mathematical Sciences, \\ University of Western Sydney, Locked Bag 1797, Penrith South DC 1797, Australia
}

\begin{abstract}
This study presents the nonlinearity and dispersion effects involved in the propagation of optical solitons which can be understood by using a numerical routine to solve the Generalized Nonlinear Paraxial equation. A sequence of code has been developed in Mathematica, to explore in depth several features of the optical soliton's formation and propagation. These numerical routines were implemented through the use with Mathematica and the results give a very clear idea of this interesting and important practical phenomenon.
\end{abstract}

Key words: Mathematica, Generalized Nonlinear Paraxial Equation, Solitons

\section{INTRODUCTION}

The field of nonlinear optics has developed in recent years as nonlinear materials have become available and widespread applications have become apparent. This is particularly true for optical solitons and other types of nonlinear pulse transmission in optical fibers. Subsequently, this form of light propagation can be utilized in the future for very high capacity dispersion-free communications. The purpose of this study is to describe the use of a very powerful tool to solve the generalized Nonlinear Paraxial equation that has stable solutions called optical solutions $^{[2]}$. The solitary wave (or soliton) is a wave that consists of a single symmetrical hump that propagates at uniform velocity without changing its form. The physical origin of solitons is the Kerr effect, which relies on a nonlinear dielectric constant that can balance the group dispersed in the optical propagation medium. The resulting effect of this balance is the propagation of solitons, which has the form of a hyperbolic secant ${ }^{[3]}$.

Nonlinear paraxial equation: The electric field $\mathrm{E}$ in an optical fiber with the dielectric constant $\varepsilon$ satisfies the Maxwell equation:

$\nabla \times \nabla \times \mathrm{E}=-\frac{1 \partial^{2}}{\mathrm{c}^{2} \partial \mathrm{t}^{2}} \mathrm{D}$

Where, $\mathrm{c}$ is the speed of light, and the displacement D $=\varepsilon \times \mathrm{E}$. By virtue of a formula from vector calculus, Eq. (1) can be written in the form:

$\nabla^{2} \mathrm{E}=-\frac{1 \partial^{2}}{\mathrm{c}^{2} \partial \mathrm{t}^{2}} \mathrm{D}=\nabla(\nabla . \mathrm{E})$

It should be noted that $\nabla \cdot \mathrm{E}$ in Eq. (2) is not zero, since $\nabla \cdot \mathrm{D}=0$. The constraint for $\mathrm{D}$ in Maxwell's equation implies that: $\varepsilon \times(\nabla \cdot E)=-(\nabla \varepsilon) \cdot E \neq 0$

This implies that Eq. (2) cannot be reduced simply to a scalar equation. For the purpose of this paper, Eq. (2) can be reduced in the sense of the asymptotic perturbation method. This is done by writing Eq. (2) in the following form:

$\mathrm{LE}=0$

Where, $\mathrm{E}$ expresses a column vector, i.e., $\mathrm{E}=\left(\mathrm{E}_{\mathrm{r}}, \mathrm{E}_{\theta}\right.$, $\mathrm{E}_{\mathrm{z}}{ }^{\mathrm{t}}$ and in the cylindrical coordinates with the $\mathrm{z}$-axis as the axial direction of the fiber, the matrix $\mathrm{L}$ consisting of the three parts $\mathrm{L}=\mathrm{L}_{\mathrm{a}}+\mathrm{L}_{\mathrm{b}}-\mathrm{L}_{\mathrm{c}}$ is defined:

$\mathrm{L}_{\mathrm{a}}=\left[\begin{array}{ccc}\nabla_{\perp}^{2}-\frac{1}{\mathrm{r}^{2}} & -\frac{2}{\mathrm{r}^{2}} \frac{\partial}{\partial \theta} & 0 \\ -\frac{2}{\mathrm{r}^{2}} \frac{\partial}{\partial \theta} & \nabla_{\perp}^{2}-\frac{1}{\mathrm{r}^{2}} & 0 \\ 0 & 0 & \nabla_{\perp}^{2}\end{array}\right]$

$\mathrm{L}_{\mathrm{b}}=\left[\frac{\partial^{2}}{\partial \mathrm{z}^{2}}-\frac{1}{\mathrm{c}^{2}} \frac{\partial^{2}}{\partial \mathrm{t}^{2}} \varepsilon\right] \cdot\left[\begin{array}{ccc}1 & 0 & 0 \\ 0 & 1 & 0 \\ 0 & 0 & 1\end{array}\right]$

$\mathrm{L}_{\mathrm{c}}=\left[\begin{array}{ccc}\frac{\partial}{\partial \mathrm{r}} \frac{1}{\mathrm{r}} \frac{\partial}{\partial \mathrm{r}} \mathrm{r} & \frac{\partial}{\partial \mathrm{r}} \frac{1}{\mathrm{r}} \frac{\partial}{\partial \theta} & \frac{\partial^{2}}{\partial \mathrm{r} \partial \mathrm{z}} \\ \frac{1}{\mathrm{r}^{2}} \frac{\partial^{2}}{\partial \mathrm{r} \partial \theta} \mathrm{r} & \frac{1}{\mathrm{r}} \frac{\partial^{2}}{\partial \theta^{2}} & \frac{1}{\mathrm{r}} \frac{\partial^{2}}{\partial \theta \partial \mathrm{z}} \\ \frac{1}{\mathrm{r} \frac{\partial^{2}}{\partial \mathrm{r} \partial \mathrm{z}} \mathrm{r}} & \frac{1}{\mathrm{r}} \frac{\partial^{2}}{\partial \theta \partial \mathrm{z}} & \frac{\partial^{2}}{\partial \mathrm{z}^{2}}\end{array}\right]$

It should be noted that these matrices imply that: 


$$
\begin{aligned}
& \mathrm{L}_{\mathrm{a}} \mathrm{E}=\nabla_{\perp}^{2} \mathrm{E} \equiv\left(\frac{1}{\mathrm{r}} \frac{\partial}{\partial \mathrm{r}} \mathrm{r} \frac{\partial}{\partial \mathrm{r}}+\frac{1}{\mathrm{r}^{2}} \frac{\partial^{2}}{\partial \theta^{2}}\right) \mathrm{E}, \\
& \mathrm{L}_{\mathrm{b}} \mathrm{E}=\left(\frac{\partial^{2}}{\partial \mathrm{z}^{2}}-\frac{1}{\mathrm{c}^{2}} \frac{\partial^{2}}{\partial \mathrm{t}^{2}} \varepsilon\right) \mathrm{E}, \\
& \mathrm{L}_{\mathrm{c}} \mathrm{E}=\nabla(\nabla . \mathrm{E})
\end{aligned}
$$

The electric field is considered as a monochromatic wave propagating along the $\mathrm{z}$-axis with the wave number $\mathrm{k}$ and angular frequency $\omega$, that is, the field $\mathrm{E}$ is assumed to be in the expansion form:

$$
\mathrm{E}(\mathrm{r}, \theta, \mathrm{z}, \mathrm{t})=\sum_{1=-\infty}^{\infty} \mathrm{E}_{1}(\mathrm{r}, \theta, \xi, \tau ; \varepsilon) \exp [\mathrm{i}(\mathrm{kz}-\omega \mathrm{t})]
$$

With $\mathrm{E}_{-1}=\mathrm{E}_{1}^{*}$ (complex conjugate) where $\mathrm{k}_{1}=\mathrm{lk}$, $\omega_{1}=1 \omega$ and the summation is taken over all harmonics generated by the nonlinearity due to the Kerr effect and $\mathrm{E}_{\mathrm{l}}(\mathrm{r}, \theta, \xi, \tau ; \varepsilon)$ is the envelope of the $\mathrm{l}^{\text {th }}$ harmonic changing slowly in $\mathrm{z}$ and $\mathrm{t}$. The slow variables $\xi$ and $\tau$ are defined by:

$$
\xi=\varepsilon^{2} \mathrm{z} \text { and } \tau=\varepsilon\left(\mathrm{t} \frac{\mathrm{z}}{\mathrm{V}_{\mathrm{g}}}\right)
$$

Where the small parameter $\varepsilon(|\varepsilon|<<1)$ expresses the order of nonlinearity (i.e., the order of the electric field) and $\mathrm{V}_{\mathrm{g}}$ is the group velocity of the wave. On this scale of the coordinates, Eq. (7) is looking at the behavior of the field in the balance between the nonlinearity and the dispersion, which results in the forming of optical solitons, confined in the transverse direction. From Eq. (6) and Eq. (7), the displacement is found by:

$D=\varepsilon \times E=\sum D_{1} \exp \left[i\left(k_{1} z-\omega_{1} t\right)\right]$

It has been shown by ${ }^{[4]}$ that $\mathrm{E}_{\mathrm{l}}(\mathrm{r}, \theta, \xi, \tau ; \varepsilon)$ can be expanded in terms of $\varepsilon$ :

$$
\mathrm{E}_{1}(\mathrm{r}, \theta, \xi, \tau ; \varepsilon)=\sum_{\mathrm{n}=1}^{\infty} \varepsilon^{\mathrm{n}} \mathrm{E}_{\mathrm{t}}^{(\mathrm{n})}(\mathrm{r}, \theta, \xi, \tau)
$$

So in order $\varepsilon$ :

$$
\mathrm{L}_{1} \mathrm{E}_{1}^{(1)}=0
$$

where, $\mathrm{L}_{1}$ is $\mathrm{L}$ with the replacements:

$$
\frac{\partial}{\partial z}=i K_{1}, \frac{\partial}{\partial t}=-i \omega_{1}, \varepsilon=\varepsilon_{1}^{(0)}
$$

Note that the operator $\mathrm{L}_{1}$ is self-adjoint, $\mathrm{L}_{1}^{+}=\mathrm{L}_{1}$, in the sense of the following inner product:

$$
(\mathrm{U}, \mathrm{V})=\int_{\mathrm{D}} \mathrm{U}^{+} \mathrm{Vds}
$$

where, $\mathrm{ds}=\operatorname{rdrd} \theta$ is the cross section of the fiber and $\mathrm{A}^{+}$is the adjoint $\mathrm{A}=\left(\mathrm{A}_{\mathrm{ij}}\right)^{+}=\left(\mathrm{A}_{\mathrm{ji}}^{*}\right)$.

In Eq. (10), there is only one bound state with the eigenvalue $K_{1}^{2}$ (i.e., $1= \pm 1$ ) and the eigen function $U$ $=\mathrm{U}(\mathrm{r}, \theta)$ called the mode function describing the confinement of the pulse in the transverse direction and, in general, consists of two parts corresponding to the right and left polarizations. Then, the solution to Eq. (10) may be written as:

$\mathrm{E}_{1}^{1}(\mathrm{r}, \theta, \xi, \tau)=\left\{\begin{array}{cc}\mathrm{u}_{1}^{(1)}(\xi, \tau) \mathrm{U}(\mathrm{r}, \theta) & \text { for } 1= \pm 1 \\ 0 & \text { for } 1 \neq \pm 1\end{array}\right\}$

Here the coefficient $u_{1}^{(1)}(\xi, \tau)$ with $u_{-1}^{(1)}=u_{1}^{(1)}$ is a complex scalar function satisfying certain equations given in the higher-order Eq. (4). From the equation $\mathrm{L}_{1} \mathrm{U}=0$, the inner product $\left(\mathrm{U}, \mathrm{L}_{\mathrm{l}} \mathrm{U}\right)=0$ gives the linear dispersion relation $\mathrm{K}_{1}=\mathrm{K}\left(\omega_{1}\right)$ :

$$
\mathrm{K}_{1}^{2}=\frac{\omega^{2}}{\mathrm{c}^{2}}\left(\mathrm{U}, \mathrm{N}_{0}^{2} \mathrm{U}\right)+\left(\mathrm{U}, \mathrm{L}_{0} \mathrm{U}\right)
$$

where, $\mathrm{N}_{0}=\left(\varepsilon_{1}^{(0)}\right)^{1 / 2}$ is the index of refraction, and have assumed the normalization for $\mathrm{U}$ by $\mathrm{U}_{\mathrm{x}}^{2}+\mathrm{U}_{\mathrm{y}}^{2}=1$.

At order $\varepsilon^{(2)}$ :

$\mathrm{L}_{1} \mathrm{E}_{1}^{2}=\mathrm{i}\left[-\frac{\partial \mathrm{L}_{1}}{\partial \omega_{1}}+\left(\frac{1}{\mathrm{~V}_{\mathrm{g}}}-\frac{\partial \mathrm{K}_{1}}{\partial \omega_{1}}\right) \frac{\partial}{\partial \omega_{1}}\left(\mathrm{~L}_{1}-\frac{\omega_{1}^{2}}{\mathrm{c}^{2}} \varepsilon_{1}^{(0)}\right)\right] \cdot \frac{\partial \mathrm{E}_{1}^{1}}{\partial \tau}$

From which $\mathrm{E}_{1}^{(2)}=0$ is obtained if $1= \pm 1$. In the case $1=1$ it is required that the inhomogeneous Eq. (14) satisfies the compatibility (or integrability) condition:

$$
\left(\mathrm{U}, \mathrm{L}_{1} \mathrm{E}_{1}^{2}\right)=0
$$

This gives the group velocity $\mathrm{V}_{\mathrm{g}}$ in terms of the linear dispersion relation Eq. (16):

$$
\frac{1}{\mathrm{~V}_{\mathrm{g}}}=\frac{\partial \mathrm{k}_{1}}{\partial \omega_{1}}
$$

For $1=1$, Eq. (14) becomes:

$\mathrm{L}_{1} \mathrm{E}_{1}^{2}=-\mathrm{i} \frac{\partial \mathrm{L}_{1}}{\partial \omega_{1}} \frac{\partial \mathrm{E}_{1}^{(1)}}{\partial \tau}=\mathrm{i} \frac{\partial \mathrm{L}_{1}}{\partial \omega_{1}} \frac{\partial \mathrm{u}_{1}^{(1)}}{\partial \tau} \cdot \mathrm{U}$

From Eq. (10) for $1=1$, the solution of Eq. (17) may be found in the form ${ }^{[1,5]}$ :

$$
\mathrm{E}^{2}=\mathrm{i} \frac{\partial \mathrm{E}_{1}^{(1)}}{\partial \tau} \cdot \frac{\partial \mathrm{U}}{\partial \omega_{1}} \mathrm{u}_{1}^{(2)} \mathrm{U}
$$


where, $\mathrm{u}_{1}^{(2)}=\mathrm{u}_{1}^{(2)}(\xi, \tau) \quad$ with $\quad \mathrm{u}_{-1}^{(2)}=\mathrm{u}_{1}^{(2)} \quad$ is a scalar function to be determined in the higher-order equation.

At order $\varepsilon_{1}^{(3)}$ :

$$
\mathrm{L}_{1} \mathrm{E}^{(3)}\left\{\begin{array}{c}
=0 \text { if } \mathrm{l} \neq \pm 1, \pm 3 \\
=-\frac{9 \omega_{1}^{2}}{\mathrm{c}^{2}} \varepsilon_{3}^{(2)} \mathrm{u}_{1}^{(1) 3}(\mathrm{U} . \mathrm{U}) \mathrm{U} \text { if } \mathrm{l}=3 \\
=-\frac{\mathrm{i} \partial \mathrm{L}_{1}}{\partial \omega_{1}} \frac{\partial \mathrm{E}_{1}^{(2)}}{\partial \tau}+\frac{1}{2} \frac{\partial^{2} \mathrm{~L}_{1}}{\partial \omega_{1}^{2}} \frac{\partial^{2} \mathrm{E}_{1}^{(1)}}{\partial \tau^{2}} \\
+\left(\mathrm{i} \frac{\partial \mathrm{u}_{1}^{(1)}}{\partial \tau}-\frac{1}{2} \frac{\partial^{2} \mathrm{k}_{1}}{\partial \omega_{1}^{2}} \frac{\partial^{2} \mathrm{u}_{1}^{(1)}}{\partial \tau}\right) \\
\times\left[\frac{\partial}{\partial \mathrm{K}_{1}}\left(\mathrm{~L}_{1}-\frac{\omega_{1}^{2} \mathrm{n}_{0}^{2}}{\mathrm{c}^{2}}\right)\right] \mathrm{U}-\left|\mathrm{u}_{1}^{(1)}\right|^{2} \\
\mathrm{u}_{1}^{(1)} \frac{\omega_{1}^{2} \varepsilon_{1}^{(2)}}{\mathrm{c}^{2}}(\mathrm{U} . \mathrm{U}) \mathrm{U}, \text { if } \mathrm{l}=1
\end{array}\right\}
$$

Where:

$$
\varepsilon_{1}^{(2)}=\sum_{11+12+13} \varepsilon_{11 / 2 / 3}^{(2)}\left(l_{i}= \pm 1\right)
$$

Note that $\varepsilon_{1}^{(2)}$ is a positive real number for the Kerr effect. From Eq. (19), one can obtain the solutions, $\mathrm{E}_{1}^{(3)}=0$ for $1= \pm 1$ or \pm 3 , and seigenmodeoes not have the eigenmode:

$$
\mathrm{E}_{3}^{(3)}=\frac{9 \omega_{1}^{2}}{\mathrm{c}^{2}} \mathrm{u}_{1}^{(1) 3} \mathrm{~L}_{3}^{-1}\left[\varepsilon_{3}^{(2)}(\mathrm{U} \cdot \mathrm{U}) \mathrm{U}\right]
$$

Which is a harmonic generated by the nonlinearity. For $1=1$, the compatibility condition is required:

$$
\left(\mathrm{U}, \mathrm{L}_{1} \mathrm{E}_{1}^{(3)}\right)=0
$$

From which the Generalized Nonlinear Paraxial equation for $u_{1}^{(1)}(\xi, \tau)$ is obtained ${ }^{[1,5]}$ :

$i \frac{\partial u}{\partial \xi}+\frac{1}{2} \frac{\partial^{2} u}{\partial \tau^{2}}+|u|^{2} u=-i \Gamma u+i \delta \frac{\partial^{3} u}{\partial \tau^{3}}$

Now the new variables and constants are introduced:

$$
\begin{aligned}
& \xi=\mathrm{z}: \tau=\mathrm{t}-\frac{\mathrm{z}}{\mathrm{v}_{\mathrm{g}}} \\
& \mathrm{u}=\frac{\omega_{0}}{2 \mathrm{k}_{0}} \sqrt[3]{\mathrm{u}_{0} \varepsilon_{0} \psi}: \frac{\partial \mathrm{k}}{\partial \omega}=\mathrm{k}^{\prime} \\
& \Gamma=\frac{\mathrm{u}_{0} \sigma}{\sqrt[3]{\mathrm{u}_{0} \varepsilon_{0}}}: \delta=\frac{\sqrt[6]{\mathrm{u}_{0} \varepsilon_{0} \mathrm{k}^{\prime \prime}}}{6 \mathrm{k}^{\prime \prime} \sqrt{2 \mathrm{k}_{0} \mathrm{k}^{\prime \prime}}}
\end{aligned}
$$

$\mathrm{k}^{\prime \prime}=\frac{\partial^{2} \mathrm{k}}{\partial \omega^{2}}: \mathrm{k}^{\prime \prime}=\frac{\partial^{3} \mathrm{k}}{\partial \omega^{3}}$

The importance of Eq. (22) is that it can be saved into normalized reference coordinates. A clear view of the evolution of the envelope along the normalized propagation path results. This will also allow us to study the different cases, such as the classical situation, where $\Gamma=0$, which results in the standard Nonlinear Paraxial equation $^{[5]}$.

Initial conditions: The solution of the Nonlinear Paraxial equation can be solved exactly by the inverse scattering method. A planar stationary light beam in a medium with a nonlinear refractive index can be described as a dimensionless form ${ }^{[6]}$.

$\mathrm{i} \frac{\partial \mathrm{u}}{\partial \xi}+\frac{\partial^{2} \mathrm{u}}{\partial \tau^{2}}+\mathrm{k}|\mathrm{u}|^{2} \mathrm{u}=0$

The method used to solve the exact inverse scattering method is applicable to equations of the type:

$\frac{\partial u}{\partial \xi} \hat{S}[u]$

where, $\hat{\mathrm{S}}$ is a nonlinear operator differential in $\mathrm{z}$, which can be represented in the form:

$\frac{\partial \hat{\mathrm{L}}}{\partial \xi}=\mathrm{i}[\hat{\mathrm{L}}, \hat{\mathrm{A}}]$

Here $\hat{\mathrm{L}}$ and $\hat{\mathrm{A}}$ are linear dif (e, ential operators containing the sought function $\mathrm{u}(\mathrm{z}, \mathrm{t})$ in the form of a coefficient. If the condition of Eq. (29) is satisfied, then the spectrum of the operator $\mathrm{L}$ does not depend on the initial values with respect to time. The reconstruction of the function $\mathrm{u}(\mathrm{z}, \mathrm{t})$ at any arbitrary instant of time is realized by solving the inverse scattering problem for the operator $\hat{\mathrm{L}}$.

The result in Eq. (27) can be verified in Eq. (29) with the operator's $\hat{\mathrm{L}}$ and $\hat{\mathrm{A}}$ taking the form:

$\hat{\mathrm{L}}=\left[\begin{array}{cc}1+\rho & 0 \\ 0 & 1-\rho\end{array}\right] \frac{\partial}{\partial \mathrm{z}}+\left[\begin{array}{cc}0 & \dot{\mathrm{u}} \\ \mathrm{u} & 0\end{array}\right], \mathrm{k}=\frac{2}{1-\rho^{2}}$

$\hat{\mathrm{A}}=-\rho\left[\begin{array}{ll}0 & 0 \\ 0 & 0\end{array}\right] \frac{\partial^{2}}{\partial \mathrm{z}^{2}}+\left[\begin{array}{cc}\frac{|\mathrm{u}|^{2}}{1+\rho} & \mathrm{iu} \mathrm{u}_{\dot{\mathrm{x}}} \\ -\mathrm{iu} \mathrm{u}_{\mathrm{x}} & \frac{-|\mathrm{u}|^{2}}{1+\rho}\end{array}\right]$

Without the loss of generality, the assumption $\mathrm{k}$ $>2$ and $\rho^{2}>0$ can be made on the above equation. As $t$ $\rightarrow \pm \infty$ of any initial condition, a finite set of solitons 
becomes present. In this problem, an analogous role is played by the particular solutions of the Nonlinear Paraxial equation ${ }^{[1,5]}$ :

$$
\begin{aligned}
\mathrm{u}(\mathrm{z}, \mathrm{t})= & 2 \eta \operatorname{sech}\left[2 \eta\left(\mathrm{z}-\mathrm{z}_{0}\right)+8 \eta \xi \mathrm{t}\right] \\
& \exp \left[\mathrm{i}\left(-2 \xi \mathrm{x}-4\left(\xi^{2}-\eta^{2}\right)\right) \mathrm{t}+\phi\right]
\end{aligned}
$$

where, $\eta, \xi, \phi, \mathrm{t}, \mathrm{z}_{0}$ are scaling parameters. This form of the solution can also be known as a soliton that has a stable formation.

The soliton Eq. (31) is the simplest representative of an extensive family of exact solutions of Eq. (27); this can be shown in an explicit form ${ }^{[1]}$. In the general case such a solution can also be called an $\mathrm{N}$-soliton solution, which depends on $4 \mathrm{~N}$ arbitrary constants, $\eta_{\mathrm{j}}$, $\xi_{\mathrm{j}}, \phi_{\mathrm{j}}, \mathrm{t}_{\mathrm{j}}, \mathrm{z}_{0 \mathrm{j}}$. However for the non-coinciding $\xi_{\mathrm{j}}$ this solution breaks into individual solitons if $\mathrm{t} \rightarrow \pm \infty$. Using this solution and beginning at the origin $\mathrm{z}=0$, $\mathrm{a}$ wave formation can be acknowledged by ${ }^{[5]}$ :

$\mathrm{u}(0,1)=\eta \operatorname{sech}\left[\mathrm{t}-\mathrm{t}_{0}\right]$

This initial condition takes $\mathrm{z}=0$, because we are investigating the effect of the coefficient on the evolution of the wave, and to do this we must start at $\mathrm{z}$ $=0$, not the usual initial condition where $\mathrm{t}=0$.

Finite difference solution: In order to compute a valid solution, Eq. (22) is converted to a finite difference equation $^{[7]}$ using $\mathrm{z}=\xi$ and $\mathrm{t}=\tau$. The time discretisation will be indicated by a $n$ superscript and the spatial position will have an associated integer subscript I. Thus $u(z, t)$ is denoted by $u_{i}^{n}$. The various $z$ values become $i \Delta z$ where $\Delta z$ is the mesh width and $i=0,1,2$, I. Similarly, the time variable becomes $\mathrm{n} \Delta \mathrm{t}$ where $\Delta \mathrm{t}$ is the time step $\mathrm{n}=0,1,2, \ldots \ldots ., \mathrm{N}$. Following a standard explicit procedure, the finite-difference version of equation (2.22) is found ${ }^{[5]}$ :

$$
\begin{aligned}
& i \frac{u_{i+1}^{n}-u_{i-1}^{n}}{2 \Delta z}+\frac{u_{i}^{n-1}-2 u_{i}^{n-1}+u_{i-1}^{n-1}}{2(\Delta t)^{2}}+\left|u_{i}^{n-1}\right|^{2} u_{i}^{n-1} \\
& =-i \Gamma u_{i}^{n-1}+i \delta \frac{u_{i}^{n+2}-2 u_{i}^{n+1}+2 u_{i}^{n-1}-u_{i}^{n-2}}{2(\Delta t)^{3}}
\end{aligned}
$$

The computer software Mathematica ${ }^{[8]}$ has all the tools to code this finite-difference equation, to solve it and to produce the output graphics in two and three dimensions.

\section{RESULTS}

This section investigates a numerical result of the Nonlinear Paraxial equation (31), for the formation of solitons with Mathematica ${ }^{[5]}$ for the following cases: a) Propagation of a simple soliton

b) Two solitons plotted on the same graph

c) Propagation of a square wave soliton

d) Propagation of two solitons at collision

A series of codes has been developed ${ }^{[1,5]}$ to assist in this process, where the initial condition in Eq. (32) is applied to the finite-difference formula Eq. (33). Figure 1a, represents the basic soliton wave, with parameters of amplitude $\eta=1$, and initial position on the t-axis, 32 . $\mathrm{t}_{0}=(\eta$ does not appear in the equation (4.1), as this is the finite difference form of equation (22)). The nature of this wave is in agreement with results found by ${ }^{[6]}$ in that it is a single pulse wave, and is stable over long distances. The effect of altering these basic parameters is to change the amplitude and lateral position of the pulse. The axes of the figures are labeled as $|\mathrm{u}(\mathrm{z}, \mathrm{t})|$ is the magnification ratio, $z$ is the lateral displacement and $\mathrm{t}$ is the time displacement.

Figure 1a, below, represents the graphical representation of the optical soliton in its simplest form. To achieve this result, all that is necessary in the programming phase is to present a simple pulse definition; pulse $2 \eta \operatorname{sech}\left[2 \eta\left(j-z_{0}\right)\right]$, where $\eta$ represents the amplitude of the wave, and $z_{0}$ is the phase constant of lateral displacement, herein referred to as the displacement constant. In this representation it is also found that it is sufficient here to define the initial pulse definition in one dimension, represented in the program as $\mathrm{j}$, referring to the $\mathrm{z}$ coordinate. Placing the amplitude constant inside the sech function serves to give the wave more body. Without its placement here would give a much narrower waveform, as shown in Fig. 1b. In this graph, the number of time divisions in the wave, specified in the program as time divisions $\tau=64$. Respectively, the number of space divisions is represented in the program by curves $=5$. Note: The wave is travelling outwards, from the origin, along the $\mathrm{t}$ axis, in these graphs having 64 divisions. To give it a lateral displacement of 32 divisions along this axis it is necessary to specify $z_{0}=32$. To give the wave a phase angle it is necessary to program the equation in two dimensions, adding to the initial pulse definition the term $8 \eta \xi \mathrm{t}$, where $\xi$ represents the time-phase constant, hereafter referred to as the phase constant. Care must be taken here, as this logic here can appear confusing, at first encounter.

Figure 2 represents two solitons, on the same graph, producing a result that shows varying amplitude and the time-shift. the wave on the left of the graph has amplitude $\eta=0.075$ and $45, z_{0}=$ while the other is $\eta=0.125$ and $25 z_{0}=$ Respectively. This Graph Serves a very important purpose in its representation here at this stage. Since it is obvious that the wave with lowest amplitude constant must be the one on the left of the graph, it establishes the above-mentioned hypothesis that the wave is moving outwards from the origin. Furthermore, the displacement of the center of the wave for the origin is established by the parameter $0_{\mathrm{z}}$. 


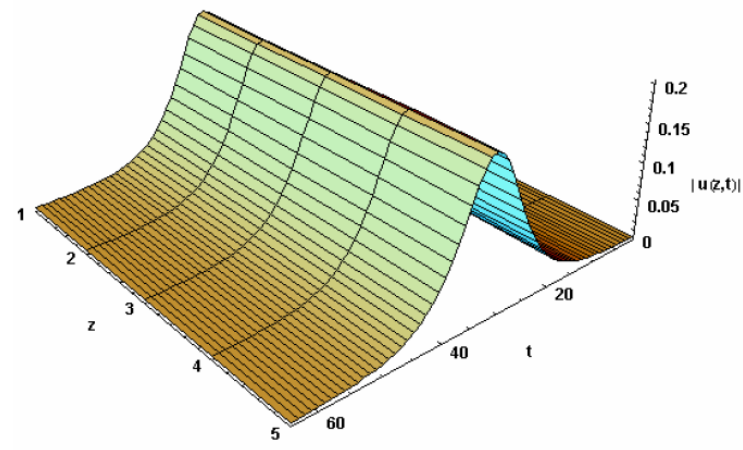

Fig. 1a: The Simple Soliton; Pulse Definition $2 \eta \operatorname{sech}[2 \eta$

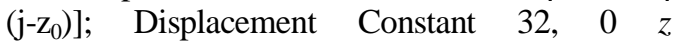
$=$ Amplitude $\eta=0.1$, and Time Divisions, $\tau=64$

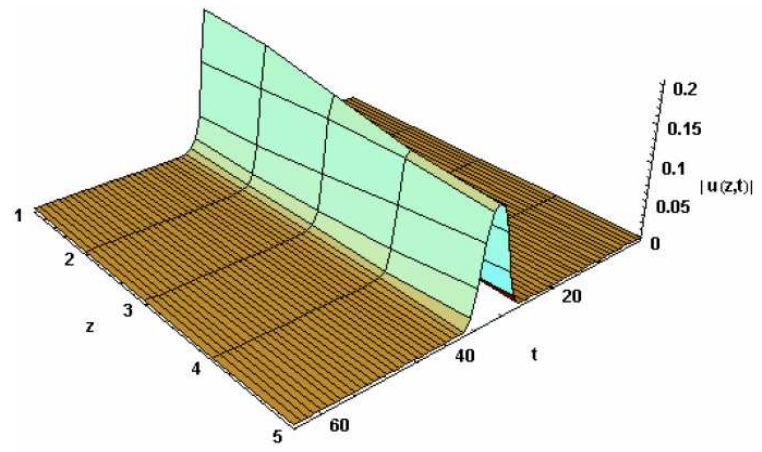

Fig. 1b: This Figures Shows the Effect of Excluding the Amplitude Constant $\eta$ from the Pulse Definition

Figure 3 represents a square wave soliton. This is the next step in the natural progression towards programming solitons in a collision. After producing the wave in Fig. 2, the program is expanded to accommodate the second variable, $t$ represented in program code by $\mathrm{k}$. This is more fully explained in the lead up to Fig. 4. This square wave is produced by using a value of $\eta=0$. 1 , where the time divisions have been expanded to $\tau=128$, and the program has been coded for two variables. This is the input format of some soliton waves as found in ${ }^{[9,10]}$ though here the wave is frozen in its square form. The appearance of the square wave is a very important point in optical solitons evolution, along the path of its rising coefficient, as this marks the point in the development of the optical soliton where, upon continuing to raise the amplitude coefficient $\eta$, the property of solitons selffocusing ${ }^{[5]}$ begins.

Figure 4 represents a soliton wave with a phase angle. This represents the first stage in the operation to show two solitons in a collision. The first task is to produce a soliton with a phase angle. As discussed above in (a), this involves the introduction of the second variable, $\mathrm{t}$ represented in the coding as the $\mathrm{k}$. The pulse definition is firstly expanded to become pulse $=2 \eta \operatorname{sech}\left[2 \eta\left(j-z_{0}\right)+8 \eta \xi t\right]$, where, $\xi$ represents the phase angle coefficient, set for Fig. 4 at $\xi=-0.07$.

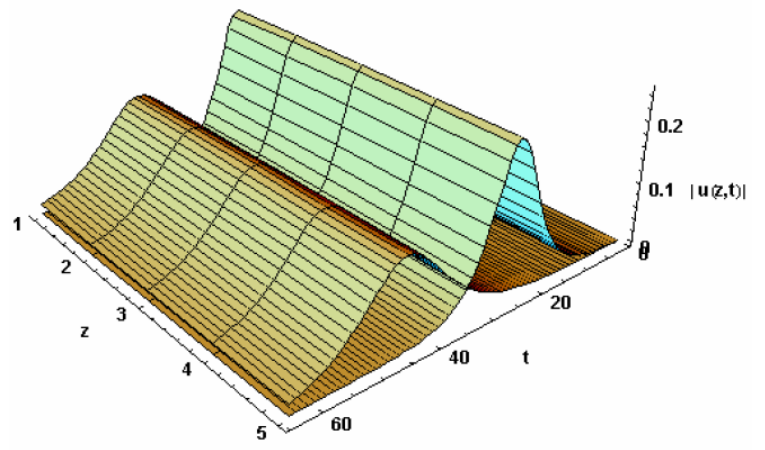

Fig. 2: Two Solitons with Amplitude at $\eta=0.25$ and $\eta=0.1$ Respective to $38 \mathrm{z}_{0}=$ and $32 ; \mathrm{z}_{1}$ $=$ Plotted on the Same Graph, to Illustrate the Difference Made by Varying the Amplitude and Lateral Displacement

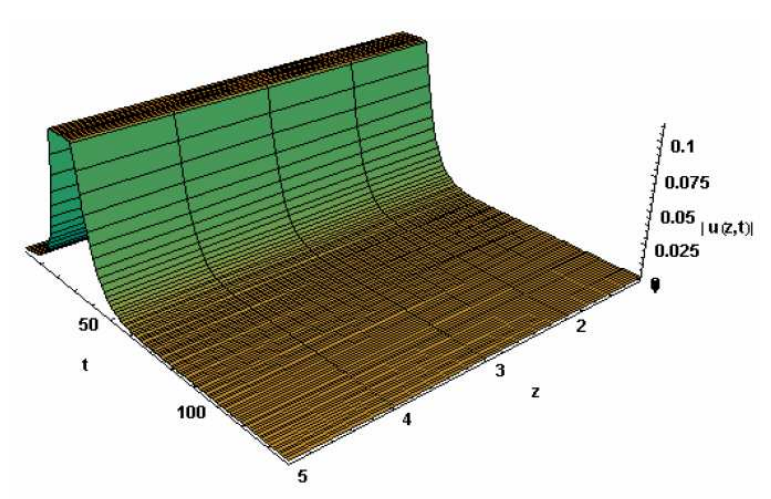

Fig. 3: Square Wave Soliton.

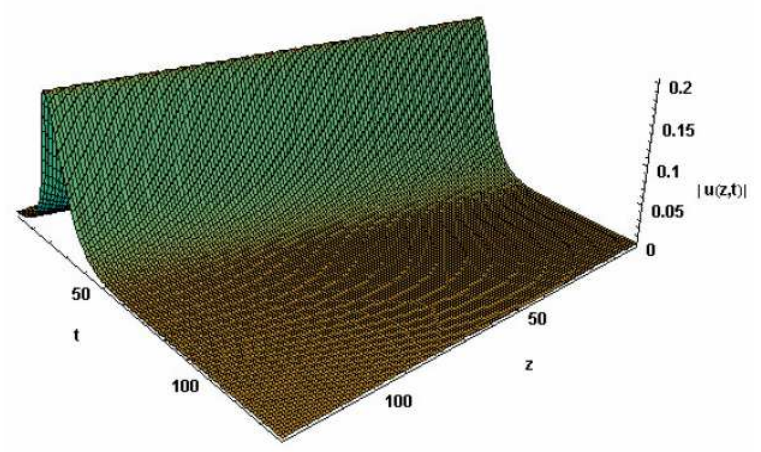

Fig. 4: Here the Program has been Allowed to set the Scales Automatically, at $0-60$, to Accommodate the Square Array in Order that the Program May Successfully Compile.

The program is then re-coded to accommodate two variables. In this graph amplitude constant $\eta$ is set at 0.1 , and displacement constant $64 \mathrm{z}_{0}=$ and time the divisions to $\tau=128$. To accommodate this it is also necessary to allow the program to automatically fix both scales for $\mathrm{z}$ and $t$ at $0-60$. The result is shown in Fig. 4. 


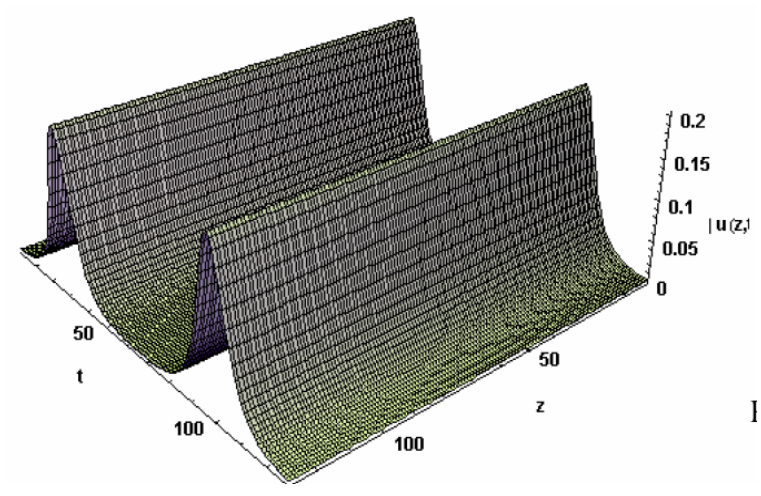

Fig. 5: Two Solitons Following in Phase

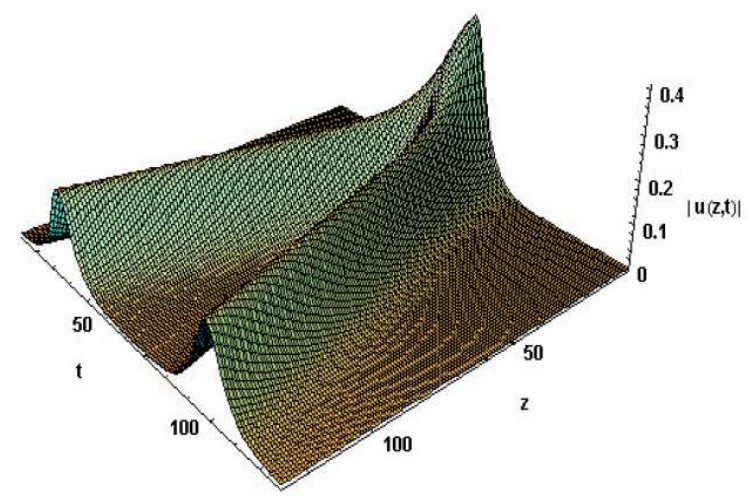

Fig. 6: Two Solitons in Early Stage of Collision.

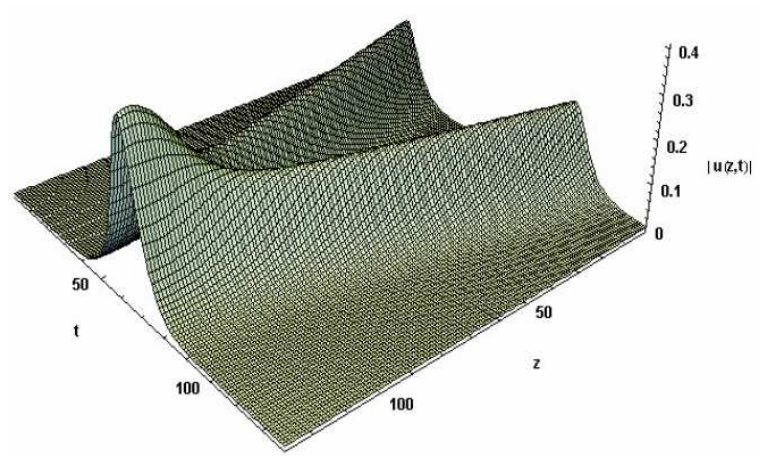

Fig. 7: Two Solitons Set at $z_{0}=100$ and $z_{1}=30$.

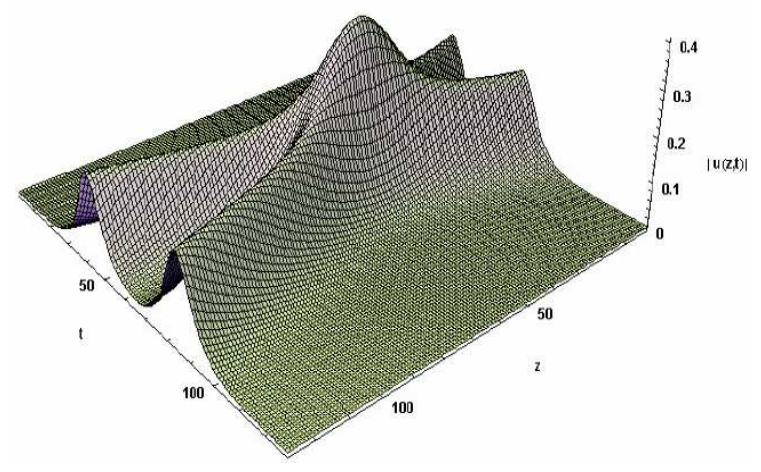

Fig. 8: The Final Effect for the Soliton Collision with $\mathrm{z}_{0}=80$ and $\mathrm{z}_{1}=50$.
Continuing with this progression, to investigate graphically the collision of solitons, the next step is to present two soliton waves following in phase. The process continues to the next step being the introduction of a second set of terms to the pulse definition, added to the first. This now becomes:

pulse $=2 \eta \operatorname{sech}\left[2 \eta\left(\mathrm{z}-\mathrm{z}_{0}\right)+8 \eta \xi_{0} \mathrm{t}\right]$,

$+2 \eta \operatorname{sech}\left[2 \eta\left(z-z_{1}\right)+8 \eta \xi_{1} t\right]$

where, $\xi_{0}$ and $\xi_{1}$ are the respective phase constants, here both set at 0 , to produce parallel waves. The displacement constants are set at $\eta_{0}=100$ and $\eta_{1}=30$.

Figure 6, represents two solitons in phase, the displacement constants are set at $\mathrm{z}_{0}=30$ and $\mathrm{z}_{0}=100$. The phase constants are set at $\xi_{1}=0.07$ and $\xi_{2}=-0$. 07 . The time divisions constant are set at $\tau=128$. This shows the two solitons, in opposing phases, meeting at the rear edge of the plot, the collision process running from the rear to the front of the curve when presented in this manner. This again follows the hypothesis set at the beginning, where the waves run outwards from the origin.

Figure 7, below represents two solitons in phase, the later displacement constants are set at $\mathrm{z}_{0}=100$ and $\mathrm{z}_{1}=30$. The phase constants are set at $\xi_{1}=0.07$ and $\xi_{2}=-0$. 07. The time divisions constant are set at $\tau=128$. Figure 7 shows the two solitons, in opposing phases, meeting at the near edge of the plot, the collision process running from the rear to the front of the curve when presented in this manner.

Figure 8, below represents the midpoint for the collision, is programmed by setting the displacement constants at $\mathrm{z}_{0}=80$ and $\mathrm{z}_{1}=50$. The phase constants and $\tau$ remains at the values used in Fig. 6 and Fig. 7 .

\section{CONCLUSION}

The formation and propagation of solutions have been observed and understood in this paper by implementing a numerical routine to solve the Nonlinear Paraxial equation ${ }^{[5]}$. A sequence of code has been developed to explore in depth several features of the soliton's formation and propagation. This paper investigated changes in the solutions of the nonlinear paraxial equation when the parameters $\eta, \xi, \phi, t, z_{0}$ are modified in the following way. The parameter $\eta$ has most noticeable effect on the amplitude of the wave.

When $\eta>0$. 1 , this condition usually encountered a square wave. When $\eta<0.1$, a normal plane soliton is resulted. Changing the inequality sign has no noticeable effect. The parameter $\xi$ gives the wave a phase angle. This phase angle becomes noticeable above 0.001 .

A phase angle above 0.1 tends to separate the soliton solution into a row of peaks. The results were found to be most useful at a phase angle of 0.07 . The direction of the phase changes with the sign. The 
parameter $t_{0}$ moves the soliton peak back and forth along the t-axis. Finally the timer divisions, on the same scale as, $\mathrm{t}_{0}$ are constant in the Mathematica $\operatorname{program}^{[1]}$. This constant investigates the spread of the graph along the t-axis. This constant was shown to be most useful when the time divisions were set at 64. A time division of 128 was used in this paper at the stage soliton collisions.

Nonlinearity and dispersion effects involved in the propagation of optical solitons have been presented in graphical form, using a numerical routine for Mathematica. This program can be used extensively to study other combined effects of pulse propagation in nonlinear dispersive media. The results displayed in this study give a very clear idea of this interesting and important practical phenomenon.

\section{REFERENCES}

1. Osman, F. 1998. PhD Thesis, University of Western Sydney.

2. Drazin, G. And Johnson, R S., 1990. Solitons: An Introduction, Cambridge University Press.

3. Whitman, G. B., 1974. Linear and Nonlinear Waves, New York: Wiley.
4. Hasegawa, A., 1989. Optical Solitons in Fibers, Springer-Verlag, Berlin.

5. Osman, F., Castillo, R. And Hora, H., 2000. Focusing and Defocusing of the Nonlinear Paraxial Equation at Laser Plasma Interaction, Laser \& Particle Beams 18, 73.

6. Zakharov, V. E. And Shabat, A. B., 1972. Exact theory of two-dimensional selffocusing and onedimensional selfmodulation of waves in nonlinear media, Soviet Physics JETP, 34, 62.

7. Smith, G. D., 1987. Numerical Solution of Partial Differential Equations: Finite Difference Methods, Oxford Applied Mathematics and Computing Science Series, 3rd edition.

8. Wolfram, S., 1991. MATHEMATICA: A System for Doing Mathematics by Computer, 2nd edition Addison-Wesley.

9. Haus, H. A., 1981. Optical Fiber Solitons, Their Properties and Uses, Proceedings of the IEEE. Vol. 81. No. 7.

10. Haus, H. A., 1993. Molding light into solitons, IEEE Spectrum. 